\title{
The cell morphogenesis ANGUSTIFOLIA (AN) gene, a plant homolog of CtBP/BARS, is involved in abiotic and biotic stress response in higher plants
}

Emma W Gachomo ${ }^{1,2}$, Jose C Jimenez-Lopez ${ }^{3}$, Sarah R Smith ', Anthony B Cooksey ${ }^{1}$, Oteri M Oghoghomeh', Nicholas Johnson ${ }^{1}$, Lamine Baba-Moussa ${ }^{4}$ and Simeon O Kotchoni ${ }^{1,2^{*}}$

\begin{abstract}
Background: ANGUSTIFOLIA (AN), one of the CtBP family proteins, plays a major role in microtubule-dependent cell morphogenesis. Microarray analysis of mammalian AN homologs suggests that AN might function as a transcriptional activator and regulator of a wide range of genes. Genetic characterization of AN mutants suggests that AN might be involved in multiple biological processes beyond cell morphology regulation.

Results: Using a reverse genetic approach, we provide in this paper the genetic, biochemical, and physiological evidence for ANGUSTIFOLIA's role in other new biological functions such as abiotic and biotic stress response in higher plants. The T-DNA knockout an-t1 mutant exhibits not only all the phenotypes of previously described angustifolia null mutants, but also copes better than wild type under dehydration and pathogen attack. The stress tolerance is accompanied by a steady-state modulation of cellular $\mathrm{H}_{2} \mathrm{O}_{2}$ content, malondialdehyde (MDA) derived from cellular lipid peroxidation, and over-expression of stress responsive genes. Our results indicate that ANGUSTIFOLIA functions beyond cell morphology control through direct or indirect functional protein interaction networks mediating other biological processes such as drought and pathogen attacks.

Conclusions: Our results indicate that the ANGUSTIFOLIA gene participates in several biochemical pathways controlling cell morphogenesis, abiotic, and biotic stress responses in higher plants. Our results suggest that the in vivo function of plant ANGUSTIFOLIA has been overlooked and it needs to be further studied beyond microtubule-dependent cell morphogenesis.
\end{abstract}

Keywords: Angustifolia, Cell morphogenesis, Arabidopsis thaliana, Abiotic stress, Biotic stress, T-DNA knockout mutant

\section{Background}

In plant cells, the overall developmental fate, whether it be height, fitness, perception of light, the efficiency of gas exchange or the response to both abiotic and biotic stress conditions, depends ultimately on well programmed cellular morphology, size, expansion, number of cellular constituents and a proper coordination of the cells. The overall rate of cell division, elongation, and cell communication contributes to the final shape of the

\footnotetext{
* Correspondence: simeon.kotchoni@rutgers.edu

'Department of Biology, Rutgers University, 315 Penn St, Camden, NJ 08102, USA

${ }^{2}$ Center for Computational and Integrative Biology, 315 Penn St, Camden, NJ 08102, USA

Full list of author information is available at the end of the article
}

cells [1,2]. In order to understand plant growth development and improve plant response to a wide range of environmental stresses it is imperative to uncover the genetic interactions and biochemical mechanisms that govern biological processes of cell shape/morphology at different stages of development. Although progress has been made in defining genetic interactions controlling the morphology of specific cell types such as trichomes (leaf hairs) and root hairs [3,4], the cellular mechanisms controlling plant response to a wide range of stress conditions remain elusive.

Recent genetic studies of Arabidopsis MICROTUBULE associated mutants including ANGUSTIFOLIA $(A N)$ and $Z W I C H E L(Z W I)$ revealed widespread cell morphological 
defects [5-7]. Several mutations of the genes affecting leaf morphology have been reported. However, the mutation affecting the ANGUSTIFOLIA gene was shown to result in narrow cotyledons, narrow rosette leaves, twisted seed pods (siliques) [8], and less-branched trichomes [5], suggesting that the $A N$ gene might play a role in leaf blade development. The narrow-leaf mutant, angustifolia (an), was originally isolated from irradiated seeds [8] and this mutation was later used as a visible marker for genetic mapping of ANGUSTIFOLIA [9]. To understand the functions of the $A N$ gene, $A N$ orthologs from various plant species including Arabidopsis, Japanese morning glory, rice, moss, and liverwort, have been recently studied $[10,11]$. Previous sequence comparison studies demonstrated that the plant $A N$ gene encodes a protein related to $\mathrm{C}$-terminal binding protein/brefeldin A ADP-ribosylated substrate (CtBP/BARS) with an important role in animal development $[12,13]$, and an encoded protein thought to repress transcription in a manner similar to that of animal CtBPs $[6,10]$. However, its function has been confirmed to be distinct from that of animal CtBP [14]. All plant AN proteins have LxCxE/D and NLS motifs that are not found in animal CtBPs $[6,10]$. Moreover, no region corresponding to the long C-terminus of the plant $A N$ genes has been detected in animal CtBPs; therefore, the $\mathrm{C}$-terminal region is thought to be related to a plant-specific function of $A N$ genes [10]. This suggests that the plant AN proteins may share some evolutionarily conserved functions with invertebrate and vertebrate CtBPs but also possess some unique functions.

So far, there is no other reported biological function of ANGUSTIFOLIA beyond the microtubule cytoskeleton mediated cell morphogenesis in plants. Meanwhile, a transcriptional role of ANGUSTIFOLIA similar to that of the invertebrate and vertebrate CtBP family proteins has been suggested on the basis of microarray analysis of transcription in angustifolia mutant background [10]. This analysis suggests that the $A N$ gene might regulate gene expression as a transcriptional repressor. In addition, the microarray analysis has indicated that several genes were expressed at higher levels in angustifolia mutant plants than in wild type [10], suggesting that ANGUSTIFOLIA may regulate leaf morphogenesis and other biological processes (i.e., association with microtubule cytoskeleton and by transcriptional regulation). In contrast, recent findings suggest that ANGUSTIFOLIA functions outside the nucleus to control cell morphogenesis [15]. This intriguing finding [15] and previously reported data could co-exist if ANGUSTIFOLIA employs two different molecular mechanisms: one to control cell morphology and the other, biological functions, respectively. We therefore checked this work for other possible biological functions of ANGUSTIFOLIA that have not been reported in plants so far. ANGUSTIFOLIA is an evolutionarily conserved protein representing a perfect model to study functional regulatory network across species.

In this work, we used a reverse genetics approach to examine and characterize a SALK_T-DNA knockout angustifolia mutant (an-t1) with respect to a wide range of biological phenotypes. Particular attention was paid to newly identified phenotypes. Our data reveal for the first time, new biological functions for ANGUSTIFOLIA in plant response to both abiotic and biotic stress conditions. The newly identified phenotypes associated with ANGUSTIFOLIA knockout mutation is involved in ROS $\left(\mathrm{H}_{2} \mathrm{O}_{2}\right)$ and stress responsive gene regulation mediating environmental stress response in higher plants. This phenotypic observation suggests reconsidering the role of ANGUSTIFOLIA beyond cell morphogenesis control.

\section{Results}

\section{Molecular characterization of an-t1 knockout mutant}

ANGUSTIFOLIA (AN) is known to regulate polarized expansions of leaf cells and leaf hair (trichome) branching via the microtubule cytoskeleton [6,10]. In this study, we further investigate the role of ANGUS TIFOLIA in other biological processes. We employed a reverse genetic approach using the Arabidopsis T-DNA SALK lines mediating loss of function of $A N$ gene to examine new $a n$-knockout phenotypes. The SALK_026 489 (an-t1) line harboring a T-DNA insertion in the first intron of the $A N$ gene (Figure 1A) was selected and confirmed as null mutant with loss of AN function. We additionally confirmed the location of the T-DNA using the T-DNA-specific oligonucleotide primer LB1 and the $A N$-specific primer Salk_026489-R and examined the $A N$ mRNA transcript levels in wild type and an-t1 mutant using RT-PCR. As shown in (Figure 1B), we confirmed that the T-DNA insertion caused a knockout of $A N$ gene in an-t1 mutant. Up to date, a T-DNA knockout line of $A N$ gene had not been characterized. We next proved that the an-t1 mutant is really an angus tifolia null mutant, by comparing its phenotype to previously well characterized angustifolia null mutants. When compared to the wild type (Figure 1C), the homozygous an-t1 mutant displayed a typical phenotype with a reduced trichome branch number (Figure 1D) -- identical to all previously reported an-null mutants $[6,10]$. The $a n-t 1$ is a recessive mutant, and the progeny of the heterozygous plants segregated with the expected 3:1 wild type:mutant Mendelian ratio (110 wild types to 36 an-t1 mutants). We next confirm that the an-t1 reducedtrichome-branch phenotype was indeed caused by the described T-DNA mutation on the ANGUSTIFOLIA gene; we performed a complementation test by crossing our an-t1 with a previously characterized angustifolia 


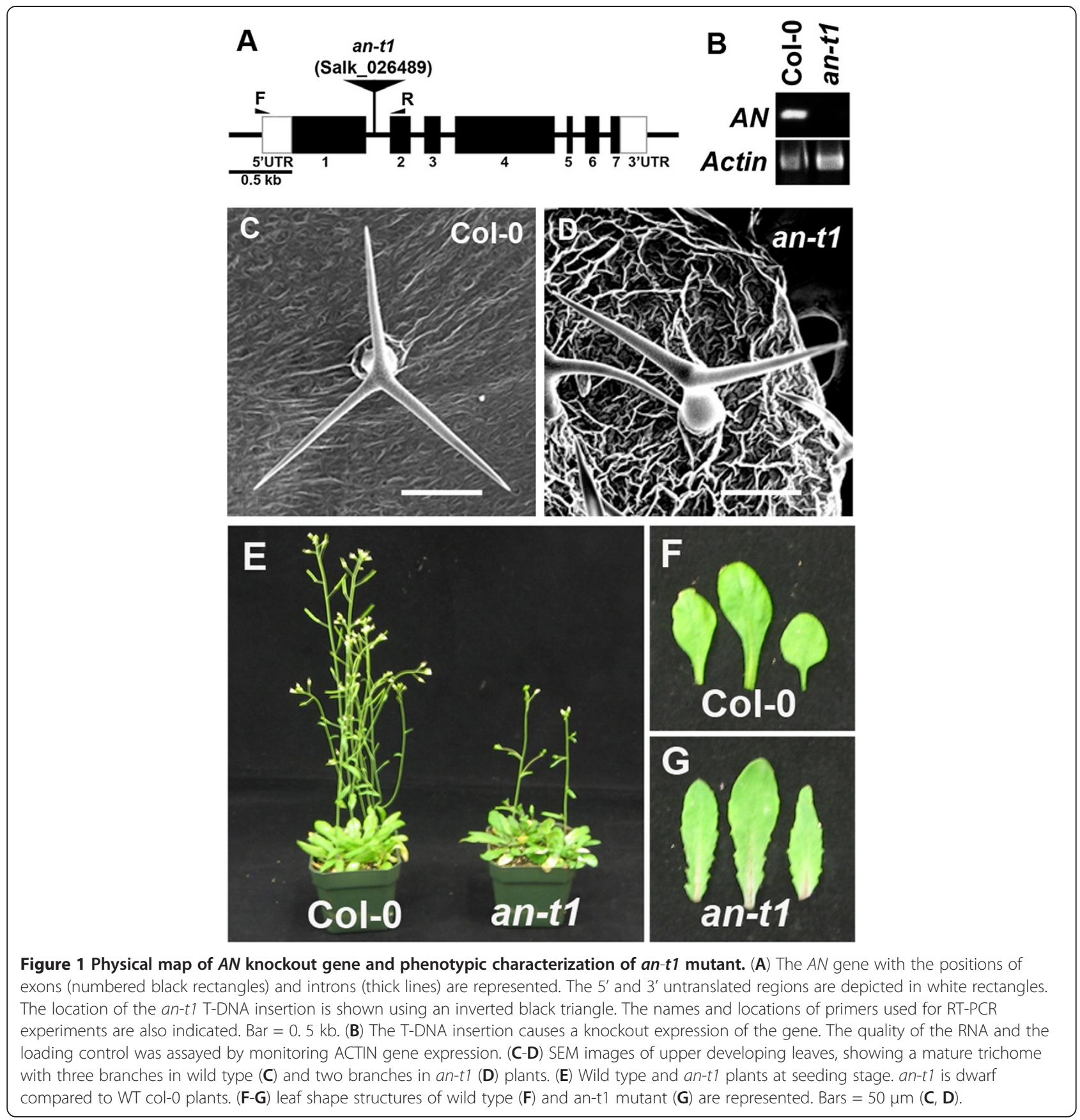

allele (an-1) [15], yielding $100 \%$ ( $\mathrm{n}=18$ seeds) of F1 progeny with reduced trichome branch numbers.

As expected, the mutation also causes a general defect in cell expansion, leading to dwarfism, (dwarf mutants) (Figure 1E), narrower leaf shapes and thickness of leaves (characteristics of all an-null mutants) in homozygous an- $t 1$ compared to the wild type as expected (Figure 1F, G). Interestingly, we noticed a significant delay in flowering time of an-t1 mutants demonstrated by a higher number of rosette leaves $(15 \pm 0.8, \mathrm{n}=10)$ compared to the wild type $(10 \pm 0.5, \mathrm{n}=12)$ at bolting time.
The loss of AN function is known to affect various organs leading to twisted seed pods (siliques). As expected, the $a n-t 1$ siliques were twisted compared to the wild type (Figure 2A,B). The mutation caused a significant seed maturation delay (Figure 2C-E). The twisted shape of $a n-t 1$ siliques was due to an irregularity in the seeds' formation (presence of gaps in the seed pods) in the an-t1 siliques compared to the wild type (Figure 2D,E). The mutation leads to a reduced number of seed pods per plant when compared to the wild type (Figure 2F). 

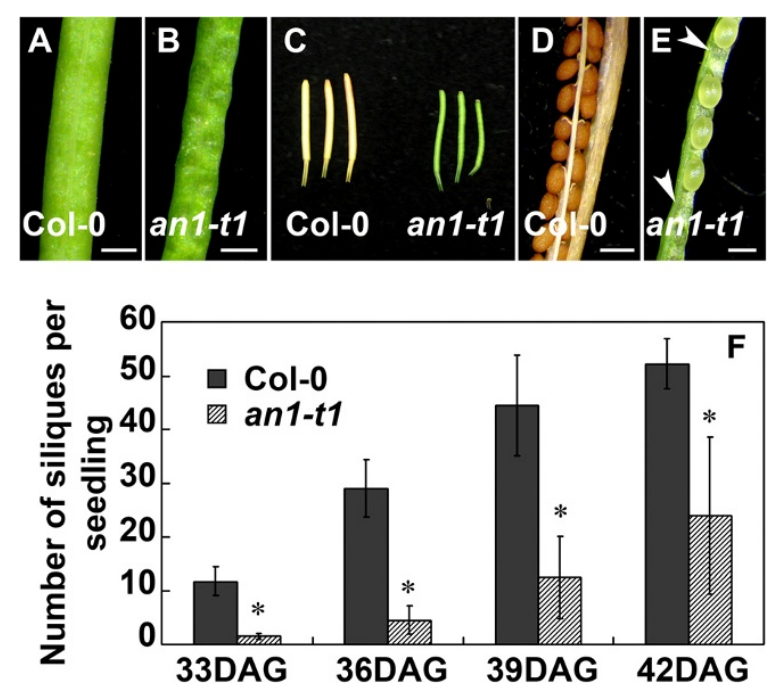

Figure 2 AN knockout gene causes irregular seed pods, delay seed maturation and reduced silique number. (A-B) Seed pod pattern of wild type (A) and an-t1 (B) is represented. (C) At the same day after germination, the silique of wild type is maturing faster than that of an-t1. (D-E) At the same day after germination, the seeds of wild type are regularly arranged in the pod and maturing faster (D) than the seeds of an-t1 with irregular arrangement (having gaps: see arrows) and still green (E). (F), The total number of siliques per plant is higher in wild type than in an-t1. Asterisks indicate significant differences between mutant and wild type. ${ }^{*} P<0.05$, Student's t test.

We next assessed the degree of sequence conservation of AN across monocot and dicot plants including the newly sequenced plant genomes. Our data revealed a high amino acid sequence identity of AN proteins across monocot and dicot plants (Additional file 1: Figure S1), indicating a functional conservation of $\mathrm{AN}$ across plant species.

\section{Novel morphological phenotype of angustifolia null mutant}

ANGUSTIFOLIA is not essential for cell viability, as an-t1 mutant is perfectly able to grow and produce seeds (Figure 1E). However, our results indicate that ANGUSTIFOLIA appears to be important to other up to now unknown interesting cell morphological phenotypes. We found an additional dark-grown phenotype associated to $a n-t 1$ mutant ( $\mathrm{n}=36$ seedlings) (Figure 3 ). The enhanced cotyledon petiole elongation has only been reported in wave and arp $2 / 3$ mutants [16,17]. We found that dark grown an-t1 plants display a strong and significantly different hypocotyl and petiole phenotype (Figure 3A,C) compared to wild type ( $\mathrm{n}=36$ an-t1 seedlings and $n=24$ WT seedlings) (Table 1 ). We checked the phenotype in the presence and absence of sucrose to rule out the effect of sugar. Our data indicate that the an-t1 dark-grown phenotype is not a result of sugar

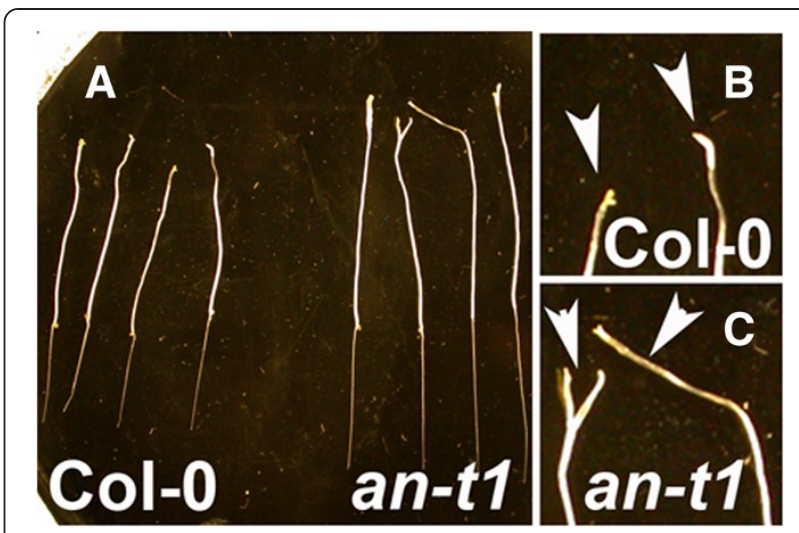

Figure 3 ANGUSTIFOLIA functions in light and dark-grown seedlings. (A-C) 7 day-after germination (DAG) of dark-grown wild type and an-t1 seedlings (A). (B) High magnification of dark-grown wild type shoots, (C) High magnification of dark-grown an-t1 shoots. Arrow heads indicate the dark-grown hypocotyls and petiole phenotype in $\mathrm{WT}$ and an- $\mathrm{t} 1$ respectively.

effect. In addition, the progeny of heterozygous WT plants segregated with the expected 3:1 wild type:mutant Mendelian ratio under dark-grown conditions, and our complementation test (an-t1 $\mathrm{x}$ an-1) gives a $100 \%(\mathrm{n}=$ 14 seeds) of F1 dark-grown phenotypic population. The enhanced elongation of petiole in dark-grown an-t1 seedlings suggests that ANGUSTIFOLIA negatively regulates growth in the petiole elongation. To be sure that this new phenotype is not restricted to our an-t1 mutant, we checked and confirmed this new light/dark phenotype in previously reported angustifolia null mutants.

The cotyledon petiole and root phenotype of the an-t1 mutant under dark grown conditions resembles the phenotype of wave mutants, which are associated with actin cytoskeleton defects [17]. We therefore hypothesized that ANGUSTIFOLIA might regulate petiole shape and root elongation through actin cytoskeleton networks. To test our hypothesis, we analyzed the growth of wild type and an-t1 mutants under latrunculin B (LatB), an actin filament depolymerization drug. Both

Table 1 Dark-grown hypocotyl and petiole phenotype of WT and angustifolia mutants

\begin{tabular}{lll}
\hline Genotypes & Hypocotyl length $(\mathbf{m m})$ & Petiole \\
\hline WT (homozygote) & $22.5 \pm 0.5(n=24) a$ & - \\
\hline$W T$ (heterozygote) & $21.6 \pm 0.3(n=18) a$ & - \\
\hline$a n-t 1$ & $16.5 \pm 0.6(n=36) b$ & + \\
\hline$a n-1^{*}$ & $18.2 \pm 0.3(n=32) b$ & + \\
\hline
\end{tabular}

The numbers in the parentheses indicate the number of samples analyzed. The sign plus indicates elongate petioles elongation in the dark, while the sign minus indicates the opposite. Mean values with different letters are significantly different from each other, and mean values with the same letter in the group are not significantly different $(P<0.05) .{ }^{*}$ an-1 mutant $=$ a gift from the Tsukaya lab. 
wild type ( $\mathrm{n}=26$ seedlings) and an-t1mutants $(\mathrm{n}=30$ seedlings) are significantly affected by Lat $B$ treatment. However, we did not observe any differences in the effect of Lat B between wild type and mutant seedlings (Additional file 2: Figure $\mathrm{S} 2, \mathrm{AB}$ ), suggesting that ANGUSTIFOLIA does not regulate root elongation and cell shape through actin cytoskeleton networks. Treatment of wild type ( $\mathrm{n}=22$ seedlings) and an-t1 mutants ( $\mathrm{n}=25$ seedlings) with Taxol, a microtubule stabilizing drug, significantly affected both cytoskeleton and root elongation in an-t1 background compared to wild type (Additional file 3: Figure S3, A-C), suggesting that ANGUSTIFOLIA controls root length and cell morphology through microtubule cytoskeleton.

\section{ANGUSTIFOLIA controls other new biological processes}

Genetic and biochemical studies have demonstrated the role of ANGUSTIFOLIA in plant cell morphogenesis [6]. However, direct evidence for the role of ANGUSTI FOLIA in other in vivo biological functional processes has not been elucidated. We hypothesized that ANGUS TIFOLIA, due to its highly conserved functional status across eukaryotes, might be involved in controlling a wide range of redox regulating and abiotic/biotic related genes. Interestingly, we noticed a higher accumulation of reactive oxygen species (ROS) phenotype in an-t1 mutants compared to the wild type (Figure 4A-D). This ROS accumulation is observed in flower organs (Figure 4B), stems (Figure 4E) and in stem-branching zones (Figure 4F). The overall accumulation of ROS was two times higher in an-t1 compared to wild type (Figure 5G), indicating that ANGUSTIFOLIA negatively

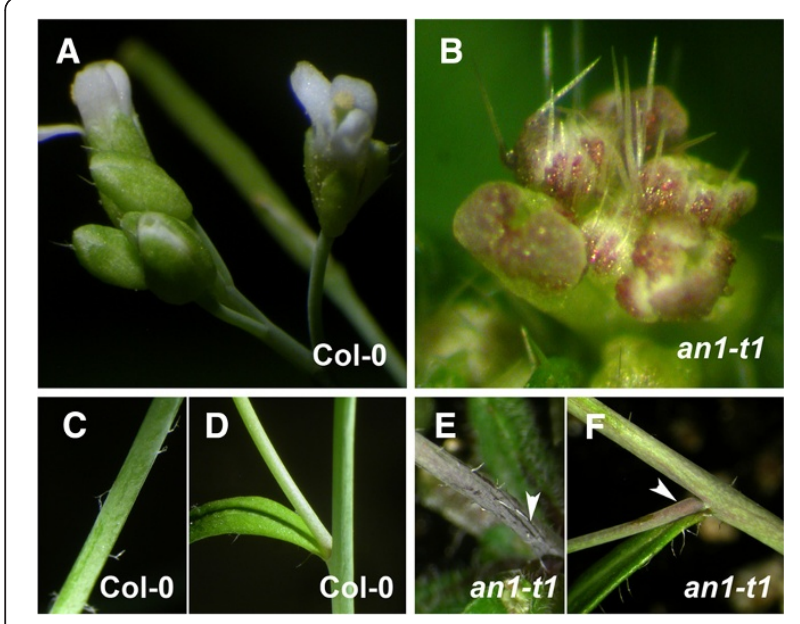

Figure 4 Mutation in $A N$ gene induces accumulation of reactive oxygen species (ROS) in plant specific plant tissues. (A-B) Strong ROS accumulation is depicted in an-t1 flowers (B) compared to wild type (A) flowers. (C-F) Accumulation of ROS in stem (E) and branches (F) of an-t1 (see arrow heads) compared to wild type stem (C) and branches (D) that are green.
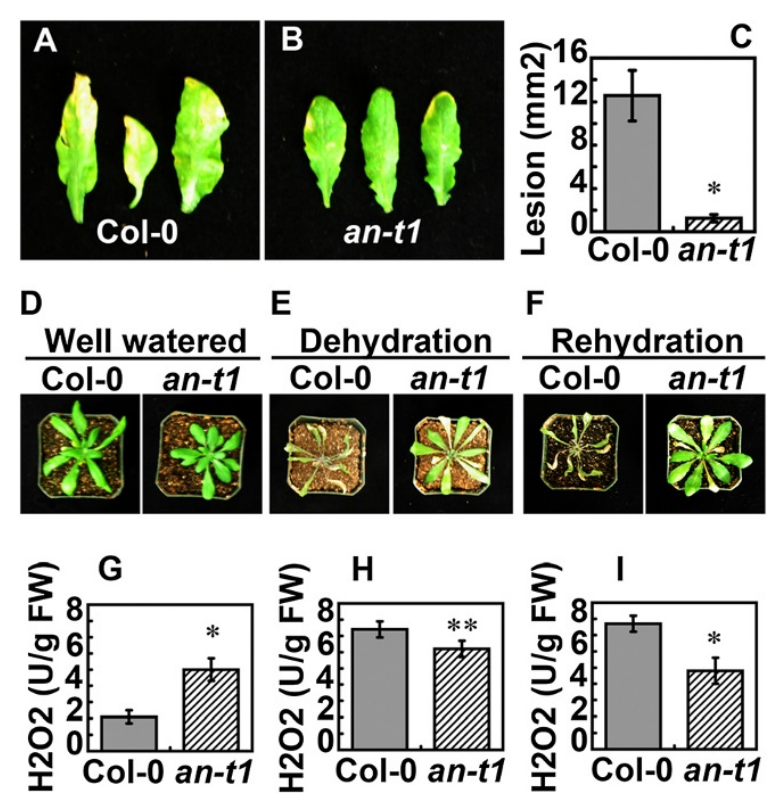

Figure 5 an-t1 seedlings cope better with drought stress and pathogen attack than the wild type. (A-C) Phenotypic characterization of wild type and an-t1 under pathogen attack. Pathogenic lesions on wild type (A) and an-t7 (B) leaves are depicted. (C) Quantitative data of pathogenic assay is represented. (D-I) Phenotypic characterization of wild type and an-t1 under well watered $(\mathbf{D})$, dehydrated $(\mathbf{E})$ and re-hydrated $(\mathbf{F})$ conditions. The $\mathrm{H}_{2} \mathrm{O}_{2}$ accumulations under well watered $(\mathbf{G})$, dehydrated $(\mathbf{H})$ and rehydrated (I) are represented. After drought stress $(\mathbf{E})$, the an-t1 was able to regain physiological functions upon re-hydration and resume normal growth, unlike wild type that withered away $(\mathbf{F}) . \mathrm{H}_{2} \mathrm{O}_{2}$ content is estimated in $\mathrm{U} / \mathrm{g} \mathrm{FW}$, where $\mathrm{U}$ represents $\mu \mathrm{mol}$, and FW represents fresh weight. Asterisks indicate significant differences between the mutant and the wild type. ${ }^{*} P<0.001$, ${ }^{* *} P<0.05$, Student's t test.

regulates the accumulation of cellular ROS. On the other hand, we hypothesized that the high ROS accumulation would predisposed an-t1 to better cope with abiotic and biotic stress conditions compared to the wild type. Indeed, $a n-t 1$ mutants ( $\mathrm{n}=10$ seedlings) were more resistant to pathogen attack than wild type ( $\mathrm{n}=10$ seedlings) (Figure 5A-C, Figure 6). To quantitatively estimate the disease resistance phenotype of $a n-t 1$, we assessed the initial rate of bacterial growth, which was found to be similar in wild type and an-t1 (Figure 6). However, at 1 to 3 days post-inoculation, the bacterial titer was approximately 5 -fold lower in an-t1 compared to the wild type (Figure 6), suggesting that an-t1 significantly suppresses the pathogen growth better than the wild type. Under drought stress conditions, an-t1 was also able to cope better than the wild type as expected (Figure 5D-I), indicating that ANGUSTIFOLIA regulates various molecular and biological processes beyond the microtubule mediated cell morphogenesis role. Figure 5(G-I) highlights the level of cellular $\mathrm{H}_{2} \mathrm{O}_{2}$ in both WT and an-t1 


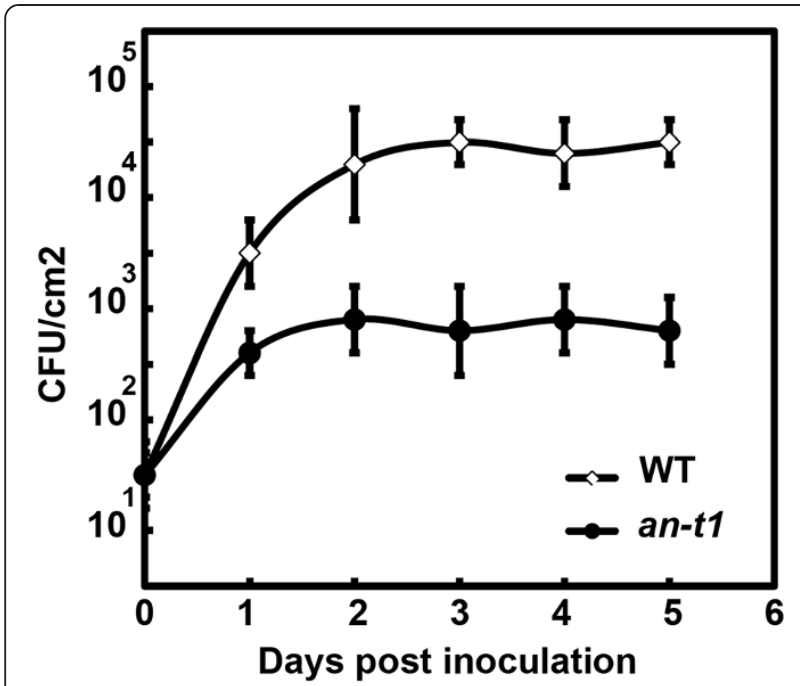

Figure 6 Responses of the wild type and an-t1 upon infection with virulent $P$. syringae pv maculicola ES4326. Leaves of 2week-old plants were inoculated with a bacterial suspension containing $10^{5} \mathrm{CFU}$ (colony forming units) $\mathrm{ml}^{-1}$. The growth curve of P. syringae pv maculicola ES4326 in wild type and an-t1 is depicted. The mean values $( \pm \mathrm{SE}$ ) of three independent experiments containing four different plants per genotype and per experiment are depicted.

mutant under normal, drought and re-watering conditions. Under this conditions, only an-t1 was able to regulate the level of endogenous $\mathrm{H}_{2} \mathrm{O}_{2}$ and therefore resume normal growth after re-watering condition compared to WT. In addition, we checked and confirmed that the drought-tolerant and pathogen-resistant phenotypes were also observed in previously reported angustifolia null mutants, indicating that these novel phenotypes are not specific to our an-t1 mutant, but rather associated to the ANGUSTIFOLIA gene in plants.

\section{ANGUSTIFOLIA regulates plant stress response through altered stress inducible gene expression}

The involvement of the ANGUSTIFOLIA gene in abiotic/biotic stress responses is particularly novel. We, therefore, investigated the accumulation of selected abiotic/biotic stress responsive transcripts in WT and an- $t 1$ to elucidate the role of ANGUSTIFOLIA in controlling stress response in higher plants (Figure 7A,B). Higher transcript levels of abiotic (drought) stress response genes was observed in the an-t1 mutant compared to WT under both control and drought stress conditions (Figure 7A,B). The osmotic/drought stress response gene, RD29A [18] was used here as the abiotic (drought) stress experimental control, while the actin gene was used as the internal loading control for the RT-PCR experiment.

It is well established that ROS accumulation in plants as a result of stress response often leads to lipid

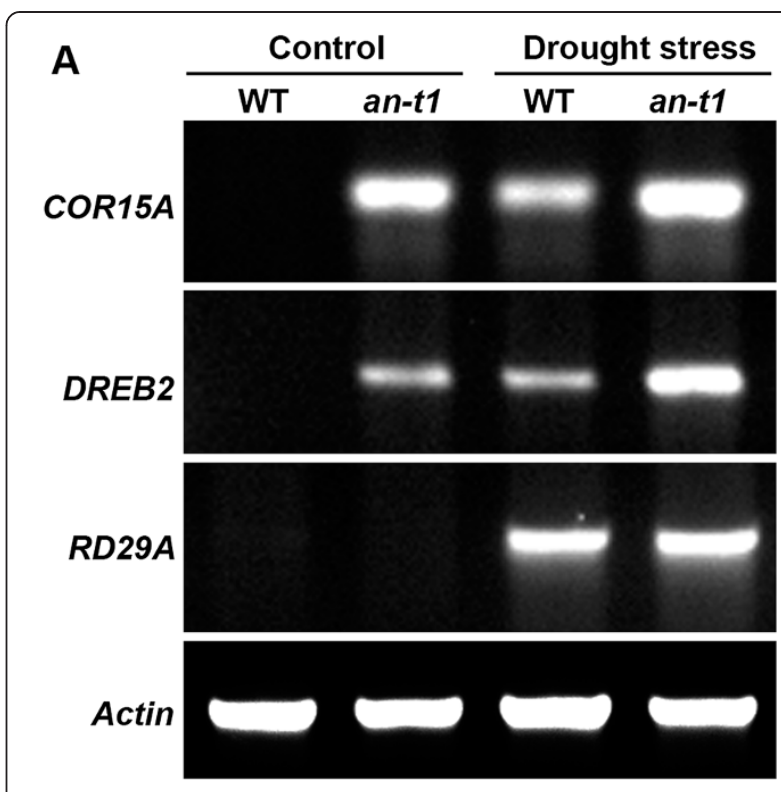

B

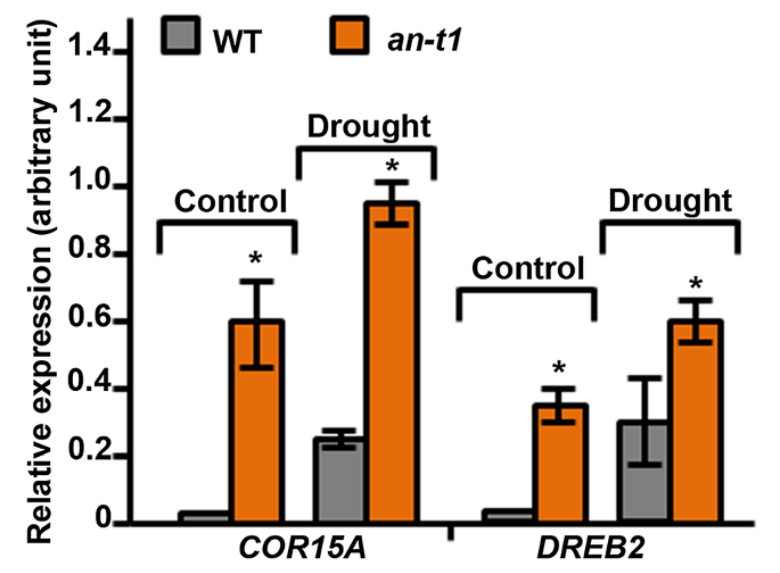

Figure 7 Stress responsive gene expression analysis in WT and angustifolia mutant backgrounds. (A): Plants were grown in soil for one week and drought stress was performed by withholding watering the plant for one week. Leaf material was collected from control and drought stress plants and used in RT-PCR expression analysis as detailed in materials and methods. RD29A (drought inducible gene) was used as positive control for stress condition, while Actin was used as internal control for RT-PCR analysis. (B): Quantification of gene expression relative to actin and RD29A transcript in control and drought stress conditions respectively. Expression data $( \pm$ SE) of three individual samples per genotype are depicted. Asterisks indicate significant differences between the mutant and the wild type ( ${ }^{*} P<0.05$, Student's $t$ test).

peroxidation [19]. To assess the role of ANGUSTI FOLIA in preventing lipid peroxidation, we analyzed the accumulation of malondialdehyde (MDA)-derived from cellular lipid peroxidation in WT and an-t1 under both control and stress conditions (Table 2). The level of MDA accumulation in $a n-t 1$ was significantly lower than that of WT under stress conditions (Table 2). 
Table 2 Malondialdehyde (MDA) derived from lipid peroxidation in WT and angustifolia mutant background under control and stress conditions

\begin{tabular}{lll}
\hline Genotypes & \multicolumn{2}{c}{ MDA (nmol/mg FW) } \\
\cline { 2 - 3 } & Control & Drought stress \\
\hline WT & $21.8 \pm 2.5(n=16) a$ & $70.5 \pm 5.3(n=16) b$ \\
\hline$a n-t 1$ & $20.5 \pm 1.8(n=22) a$ & $32.6 \pm 2.5(n=22) c$ \\
\hline$a n-1^{*}$ & $18.9 \pm 2.2(n=16) a$ & $35.2 \pm 3.8(n=16) c$
\end{tabular}

The numbers in the parentheses indicate the number of samples analyzed. Mean values with different letters are significantly different from each other, and mean values with the same letter in the group are not significantly different $(P<0.05)$. ${ }^{*}$ an-1 mutant $=$ a gift from the Tsukaya lab. Drought stress was imposed by withholding watering for one week. FW = fresh weight.

\section{ANGUSTIFOLIA regulates a wide range of protein networks}

ANGUSTIFOLIA has been postulated to function by transcriptional repression [7]. These studies raised the possibility that ANGUSTIFOLIA might also have other unrelated microtubule-associated cytoplasmic functions. Recently, ANGUSTIFOLIA has been reported to function outside the nucleus, and exclusively through membrane trafficking pathways, localizing on punctuate structures around the Golgi [15]. To further understand the function of the $A N$ gene beyond microtubuleassociated cell morphogenesis regulation, we used the latest high throughput integrated knowledge base Arabidopsis protein interaction network analysis (ANAP) tool [20] to bring insights into the functional interaction protein network of ANGUSTIFOLIA at the cellular level.

ANAP provides a far more detailed, reliable, and extensive knowledge of protein interaction networks than those produced from any single protein interaction database. Additional file 4: Figure S4 (A) shows the resulting network of 10 nodes and 11 edges, based on direct protein interactions. The ANGUSTIFOLIA query protein is marked in red in the center (Additional file 4: Figure S4, A), and each associated protein is linked by a uniquely colored line, based on the interaction detection method, and the rendering rules for the interaction detection methods (Additional file 4: Figure S4, Additional file 5: Table S1).

A more comprehensive interaction analysis using each of the 10 modes (Additional file 4: Figure S4, A) revealed an extended interaction network of 144 nodes and 234 edges (Additional file 4: Figure S4, B), where the initial 10 nodes are marked in red in the centers of the extended interactions (Additional file 4: Figure S4, B). Each associated protein is linked by a uniquely colored line, based on the interaction detection method and the rendering rules from the complete list of all interaction detection methods (Additional file 4: Figure S4, B). This interaction network (Additional file 4: Figure S4, B), which has never been reported before, sheds light into the probable role of ANGUSTIFOLIA beyond microtubule-associated cell morphogenesis control, and argues in favor of ANGUSTIFOLIA functioning in a wide range of biological processes.

\section{Discussion}

ANGUSTIFOLIA is here shown to be important not only for cell shape, trichome, and branching phenotype but also for various other biological functions including environmental stress response in plants. Our data reveals that mutations in the $A N$ gene results in delayed flowering, senescence, and reduced seed productivity. Some of these phenotypes are new and the mechanism by which ANGUSTIFOLIA regulates transition processes from the vegetative to the reproductive phase is still unknown. The role of plant ANGUSTIFOLIA at the transcriptional level is still controversial. ANGUSTI FOLIA encodes a protein with a sequence similar to CTBPs/BARS that are expressed in all organs $[6,15]$. CtBP family proteins predominantly function as transcriptional factors. We postulate that ANGUSTIFOLIA might primarily function as a transcription factor modulating the expression of a wide range of genes involved in different biological processes in plants. CTBPs in Drosophila have been shown to bind transcriptional repressors such as zinc-finger transcription factors [21]. Although, plant ANGUSTIFOLIA failed to complement Drosophila CtBP mutation, [14] uur ANAP database analysis suggested that ANGUSTIFOLIA might still act at the transcription level to control a wide range of biological processes in plants. Microarray analyses showed that transcript levels of $\sim 10$ genes in an-mutant plants were three time higher than those in the WT plants. As mentioned above, the function of ANGUSTIFOLIA has been associated only with polarized cellular growth and cell morphogenesis through microtubule cytoskeleton accessory proteins [6]. To our knowledge, this is the first time that ANGUSTIFOLIA has been shown to control environmental stress response in higher plants.

The an-t1 dark-grown phenotype (enhanced petiole elongation and a premature activation of leaf initiation (Figure $3 \mathrm{~A}-\mathrm{C}$, Table 1)) is a very new phenotype. In dark-grown WT, petiole elongation and leaf initiation are normally inhibited (Figure 3A-C, Table 1). Other previously distorted mutants that displayed this darkgrown phenotype include brk1, scar1,2,3,4 quadruple, and dis2/arpc 2 mutants [17]. The dark-grown phenotype has been also reported in the LEAFY COTYLEDON1 mutant [22]. Mutation of the LEAFY COTYLEDON1, a putative transcription co-activator, causes similar petiole and hypocotyl dark-grown phenotypes [22] suggesting that this phenotype is under a developmental-dependent regulation pathway $[17,22]$. Furthermore, the newly identified 
dark-grown phenotype indicates that ANGUSTIFOLIA negatively regulates growth in the petiole $[16,17]$.

In addition, the results presented here demonstrate that plants with a loss of function of ANGUSTIFOLIA were more tolerant to drought and pathogen attack than WT plants (Figure 5A-F). The fact that this knockout mutant line is more tolerant than the WT suggests that the expression of the $A N$ gene co-represses the abiotic and biotic associated genes. In general, no apparent phenotypic difference was observed in an-t1 and WT plants under non-stress conditions, indicating that the activity of ANGUSTIFOLIA is stress inducible.

In order to further ascertain the role of the $A N$ gene in plant stress tolerance, the expression profile of stressrelated genes was analyzed in both WT and $a n-t 1$ under control and stress conditions (Figure 7A,B). The results from the expression analysis of DREB2 (member of drought responsive element binding subfamily A-2 transcription factor family) and COR15 (cold-regulated 15-A) genes show higher transcript accumulation on an-t1 background under both control and stress conditions (Figure 7A,B). This demonstrates that the $A N$ gene negatively regulates stress-inducible genes and controls the stress response in higher plants. These findings are consistent with the suggestion that the $A N$ gene controls the stress response in plants at the transcriptional level by regulating the expression of a selected-stress-responsive and ROS-relates genes as suggested by the microarray and the interaction protein network data. The role of the $A N$ gene in stress response is further corroborated by the accumulation of higher MDA derived from cellular lipid peroxidation in WT compared to $a n-t 1$ and $a n-1$ mutants under stress conditions (Table 2).

Similar to the dehydrogenase protein superfamily, CtBP family proteins, the mammalian ANGUSTIFOLIA homologs, contain a conserved Rossman fold motif required for the binding of nicotinamide adenine dinucleotide co-factors [23]. Indeed, CtBP1 and CtBP2 have been shown to bind to $\mathrm{NAD}^{+} / \mathrm{NADH}$ redox status $[24,25]$, enhancing the interaction of CtBP with target transcription factors [25]. This redox status on modulating the repression versus activation of transcription by CtBP is an interesting aspect for future investigation. Since CtBP appears to be a redox-sensing transcriptional regulator, its activity may be modulated by the energy status of the cell during development and in diseases or abiotic stress conditions. The $A n-t 1$ knockout line displayed an elevated level of $\operatorname{ROS}\left(\mathrm{H}_{2} \mathrm{O}_{2}\right)$ under drought and pathogen attack (Figure 5G-I). This is an interesting observation because the generation of ROS and related molecules is common to both abiotic and biotic stress [26]. Both types of stress trigger the increase of $\mathrm{H}_{2} \mathrm{O}_{2}$, probably via common mechanisms. The probability of ANGUSTIFO LIA playing a role in pathogen defense and abiotic stress response has not been reported before. The stressactivated ANGUSTIFOLIA could possibly have a second role. Besides the ROS-mediated redox activity, it may catalyze reactions that can be considered as metabolic escape routes providing alternative pathways for NAD (P) $\mathrm{H}$ under various environmental stress conditions. Indeed, ANAP database analysis showed that ANGUSTI FOLIA interacts with several oxidation-reduction biological processes and abiotic and biotic stress response processes (Additional file 4: Figure S4, B, Additional file 6: Table S2) supporting the stress response phenotype observed in this study.

\section{Conclusions}

In conclusion, the present study provides a substantial body of work that demonstrates the potential of ANGUSTIFOLIA to regulate cell morphogenesis and to confer both abiotic and biotic stress tolerance through ROS-mediated redox activity. It would be interesting to investigate the ANGUSTIFOLIA knockout genes in agronomically important crops with the aim of improving crop tolerance to multiple environmental stressors. Further studies at transcriptional and proteomic levels are needed to elucidate the wide range of pleiotropic biological functions of ANGUSTIFOLIA in plants.

\section{Materials and methods}

Plant material, growth conditions, and stress treatments conditions

Arabidopsis thaliana (ecotype Col-0) and an-t1 knockout mutant (T-DNA SALK_026489) from Arabidopsis Biological Research Center (ABRC) were used throughout this work. Appropriate seeds were sown on Murashige and Skoog (MS) agar plates or soil and seedlings were allowed to grow under continuous illumination $\left(120-150 \mu \mathrm{Em}^{-2} \mathrm{~s}^{-1}\right)$ at $22^{\circ} \mathrm{C}$. Dark-growing conditions were obtained by wrapping the plates with three layers of aluminum foil and the plates were incubated under the same growth conditions. For stress conditions, seedlings were used directly from MS-agar plate and soil-grown seedlings were subjected to cytoskeleton associated drugs (LatB and Taxol) and abiotic/biotic stress treatments respectively. For dehydration stress, 2-week-old soil-grown plants were kept without watering for pre-determine time period and then re-watered according to Kotchoni et al. [19]. For pathogen infection, 2-week-old soil seedlings were infected with Pseudomonas syringae as described by Barth et al. [27] (See detail, below).

\section{Infection of plants with virulent pseudomonas syringae pv maculicola ES4326}

For pathogen infection, 2-week-old soil seedlings were infected with Pseudomonas syringae pv maculicola ES4326 as described by Barth et al. [27]. At the 
indicated time, bacterial growth in leaves was determined in $0.55 \mathrm{~cm}^{2}$ leaf discs that were extracted by macerating the discs in $300 \mathrm{ml}$ of $10 \mathrm{mM} \mathrm{MgCl}$. Serial dilutions were plated out on agar plates containing 100 $\mathrm{mg} \mathrm{ml} \mathrm{m}^{-1}$ streptomycin. The infection experiment was carried out in three independently replicate experiments.

\section{Mutant characterization and reverse transcription (RT)- PCR analysis}

T-DNA insertion in the ANGUSTIFOLIA gene was PCR-confirmed using ANGUSTIFOLIA gene specific primers (For: 5'-TACAACAACCCAAGTGGAAGA-3'; Rev: 5'-TCGAGGGCCTGATTCGTTCTT-3') and TDNA left border primer Lb: 5'-CCGTCTCACTGGTG AAAAGAA-3'. The expression of the ANGUSTIFOLIA gene in an-t1 mutant background was analyzed by extracting total RNA from the an-t1 homozygous line using TRIzol reagent (Molecular Research Center) and reverse transcribed as described previously [28]. Thereafter, the cDNA was used as template for PCR using ANGUSTIFOLIA gene specific primers. Actin2 [29] transcripts (Act2-For: 5'-GCGGATCCATGGCTGAGGCT GATGATATTCAACC-3'; Act2-Rev: 5'-CGTCTAGAC CATGGAACATTTTCTGTGAACGATTCC-3') was used as internal control.

For abiotic/biotic-gene expression analysis, genespecific primers (Table 3) were used in RT-PCR analysis to run 20 or 25 amplification PCR cycles (linear range of amplification) unless otherwise noted. The linear range of amplification was determined by running increasing cycle numbers and analyzing the amount of cDNA fragments [28]. PCR fragments were separated on 1\% agarose gels containing ethidium bromide. ACTIN gene was used as internal control [29]. For quantitative expression analysis, RT-PCR was performed in triplicate for each gene as described above and band intensities of the three replicates were respectively quantified with ImageQuant 5.0 (Amersham Biosciences). Fragments generated from ACTIN served as internal controls, while fragments of $R D 29 A$ gene served as drought stress control in both wild type and an-t1 mutant. Specifically, the mean values of pixel intensity of the bands (COR15A and $D R E B 2$ genes) were normalized against (i) ACTIN band intensity under non-stress (control) condition, and against (ii) $R D 29 A$ pixel band intensity under drought stress condition.

\section{ANAP database dependent ANGUSTIFOLIA protein interaction network}

To fully understand the role of ANGUSTIFOLIA at the molecular and cellular level in various biological processes we used the newly developed protein interaction network database ANAP (http://gmdd.shgmo.org/ComputationalBiology/ANAP/ANAP_V1.1) [20]. To construct the protein interaction network, the ANAP database tool utilized data from multiple sources, comprising both predicted interactions and experimentally tested evidence [20].

\section{Determination of flowering time}

Flowering time was assessed by counting the number of rosette leaves when flower bolts were $1 \mathrm{~cm}$ in length or when floral buds were visible at the center of the rosette as previously reported [28].

\section{Measurement of $\mathrm{H}_{2} \mathrm{O}_{2}$ content}

Rosette leaves (approximately $0.1 \mathrm{~g}$ ) were incubated under rocking condition $(250 \mathrm{rpm})$ in $3 \mathrm{ml}$ reagent $[25 \mathrm{mM}$ phosphate buffer $\mathrm{pH}$ 7.0; containing $0.05 \%$ guaiacol (Sigma) and 2.5 units $\mathrm{ml}^{-1}$ horseradish peroxidase (Sigma)] in darkness at $25^{\circ} \mathrm{C}$ for $2 \mathrm{~h}$. Absorbance of the solution was measured at $450 \mathrm{~nm}$ as described [30]. $\mathrm{H}_{2} \mathrm{O}_{2}$ concentrations were determined using a $\mathrm{H}_{2} \mathrm{O}_{2}$ standard curve, containing $5,10,25,50,75,100$, or $150 \mathrm{mM} \mathrm{H}_{2} \mathrm{O}_{2}$ (Sigma) as previously described [28].

\section{Lipid peroxidation assay}

The levels of lipid peroxidation in plant cells were assayed with the thiobarbituric acid (TBA) test, which determines the amounts of malondialdehyde (MDA) as end product of lipid peroxidation [19].

\section{Statistical analysis}

Experiments were performed at least three times. Data were expressed as mean values $\pm \mathrm{SE}$. $\mathrm{P}$ values were determined by Student's t test analysis.

Table 3 Sequences of oligonucleotide primers used for stress-responsive gene expression analysis

\begin{tabular}{llll}
\hline Sequence description & Sequence & Number of bases & AGI \\
\hline DREB2-F & TCGAGGAAAGGTTGTATGAGAGG & 23 & AT1G75490 \\
\hline DREB2-R & AGAGTCGCTGCTGCTTTTTC & 20 & AT1G75490 \\
\hline COR15A-F & ACTCAGTTCGTCGTCGTTTCTCAA & AT2G42540 \\
\hline COR15A-R & GTTGCGGCTTCTTTTCCTTTCT & 24 & AT2G42540 \\
\hline RD29A-F & ATGAGAATGGTGCGACTAA & 23 & AT5G52310 \\
\hline RD29A-R & CGTTGACCTTCCGTTGACCA & 19 & AT5G52310 \\
\hline
\end{tabular}




\section{Additional files}

Additional file 1: Figure S1. ANGUSTIFOLIA is evolutionary conserved across plant species. Comparison of ANGUSTIFOLIA full length amino acid sequences from different plant species are represented. The asterisks indicate the conserved amino acids across species. phosphorylation motifs detected in AN from Arabidopsis are indicated. Amino acids identical in all ANGUSTIFOLIA sequences are shaded in gray. At: Arabidopsis thaliana, Os: Oryza sativa, Sb: Sorghum bicolor, Zm: Zea mays, Mt: Medicago truncatula, Gm: Glycine max, AN: Angustifolia.

Additional file 2: Figure S2. The effect of latrunculin B (LatB) on wild type and an-t1 seedlings is indistinguishable. (A) Light-grown seedling phenotype of wild type and an- $t 1$ at different concentrations of LatB. (B) Root phenotype of wild type and an- $t 1$ at different concentrations of LatB. Bars $=5 \mathrm{~mm}$

Additional file 3: Figure S3. an- $t 1$ knockout mutant is more sensitive to taxol treatment than wild type. (A-B) Light-grown phenotypes of wild type (A) and an-t1 (B) at different concentrations of taxol. (C) Root phenotype of wild type and an-t1 at different concentrations of taxol. Bars $=5 \mathrm{~mm}$. Asterisks indicate significant differences between the mutant and the wild type. ${ }^{*} P<0.05$, Student's $t$ test.

Additional file 4: Figure S4. ANAP Protein interaction network generated using the ANGUSTIFOLIA protein (AT1G01510). (A) The ANAP framework of the interaction based upon the node relationship of the source database and direct interaction detection method is depicted. (B) A more comprehensive interaction of ANGUSTIFOLIA with several other proteins using a depth search mode (for indirect interaction searches) is generated.

Additional file 5: Table S1. The depth search protein interaction network generated using the ANGUSTIFOLIA protein (AT1G01510) as query. The total number of interactions detected by ANAP from experimental data and inference-based approaches supporting the interactions are depicted. The databases supporting the interactions are shown.

Additional file 6: Table S2. Selected members of Gene ontology (GO)-enriched protein interaction with Angustifolia clustered by cellular component. These proteins were selected from the extended interaction network (144 proteins) depicted in Additional file 4: Figure S4, B.

\section{Competing interests}

The authors declare that they have no competing interests.

\section{Authors' contributions}

SOK conceived the study. SOK, EWG JCJ-L and LB-M wrote the manuscript. EWG, JCJ-L, ABC, OMO, NJ and SOK performed the work and analyzed the data. SRS prepared the samples and took the SEM images and analyzed the microscopic images. All the authors approved the final manuscript.

\section{Acknowledgements}

We would like to thank Dr. Barth Carina (West Virginia University, Morgantown, W) for providing us with Pseudomonas syringae pv maculicola ES4326 strain; and Dr. Hirokazu Tsukaya (University of Tokyo, Tokyo, Japan) for providing us with the angustifolia mutant seeds (an-1). We thank Kimberlee Moran (Rutgers University, Camden) for technical assistance, proof-reading, and helpful comments on the manuscript. We acknowledged the NSF DBI-0216233 MRI grant "Acquisition of a Scanning Electron Microscope for Collaborative Use at Rutgers, Camden" for the acquisition of the Arabidopsis SEM images in this work. This work was supported by the Rutgers-University start-up funds and the RU-FAIR Mini-Grant \# 430074 to SOK.

\section{Author details}

'Department of Biology, Rutgers University, 315 Penn St, Camden, NJ 08102, USA. ${ }^{2}$ Center for Computational and Integrative Biology, 315 Penn St, Camden, NJ 08102, USA. ${ }^{3}$ Department of Biochemistry, Cell and Molecular Biology of Plants, Estación Experimental del Zaidín, Consejo Superior de Investigaciones Científicas (CSIC), Profesor Albareda 1, Granada E-18008, Spain. ${ }^{4}$ Department of Biochemistry, University of Abomey-Calavi, Cotonou, Benin.
Received: 30 August 2012 Accepted: 10 May 2013

Published: 14 May 2013

\section{References}

1. Arkebuer TJ, Norman JM: From cell growth to leaf growth: I. Coupling cell division and cell expansion. Agron J 1995, 87:99-105.

2. Maksymowych R: Cell division and cell elongation in leaf development of Xanthium pensylvanicum. Am J Bot 1963, 50:891-901.

3. Kotchoni SO, Zakharova T, Mallery EL, El-Din El-Assal S, Le J, Szymanski DB: The association of the Arabidopsis actin-related protein (ARP) $2 / 3$ complex with cell membranes is linked to its assembly status, but not to its activation. Plant Physiol 2009, 151:2095-2109.

4. Zhang C, Kotchoni SO, Samuels L, Szymanski DB: SPIKE1 signals originate from and assemble specialized domains of the endoplasmic reticulum. Curr Biol 2010, 20:2144-2149.

5. Hülskamp M, Miséra S, Jürgens G: Genetic dissection of trichome cell development in Arabidopsis. Cell 1994, 76:555-566.

6. Folkers U, Kirik V, Schöbinger U, Falk S, Krishnakumar S, Pollock MA, Oppenheimer DG, Day I, Reddy AR, Jürgens G, Hülskamp M: The cell morphogenesis gene ANGUSTIFOLIA encodes a CtBP/BARS-like protein and is involved in the control of the microtubulecytoskeleton. EMBO J 2002, 21:1280-1288.

7. Chinnadurai G: CtBP family proteins: more than transcriptional corepressors. Bioessays 2002, 25:9-12

8. Rédei GP: Single locus heterosis. Z Vererbungs/ 1962, 93:164-170.

9. Hauge BM, Hanley SM, Cartinhour S, Cherry JM, Goodmann HM, Koornneef M, Stam P, Chang C, Kempin S, Medrano L, Meyerowitz EM: An integrated genetic/RFLP map of the Arabidopsis thaliana genome. Plant J 1993 3:745-754

10. Kim GT, Shoda K, Tsuge T, Cho KH, Uchimiya H, Yokoyama R, Nishitani K, Tsukaya H: The ANGUSTIFOLIA gene of Arabidopsis, a plant CtBP gene, regulates leaf-cell expansion, the arrangement of cortical microtubules in leaf cells and expression of a gene involved in cell-wall formation. EMBO J 2002, 21:1267-1279.

11. Cho K, Shindo T, Kim G, Nitasaka E, Tsukaya H: Characterization of a member of the AN subfamily, IAN, from Ipomoea nil. Plant Cell Physiol 2005, 46:250-255.

12. Schaeper U, Boyd JM, Verma S, Uhlmann E, Subramanian T, Chinnadurai G: Molecular cloning and characterization of a cellular phosphoprotein that interacts with a conserved C-terminal domain of adenovirus E1 A involved in negative modulation of oncogenic transformation. Proc Natl Acad Sci USA 1995, 92:10467-10471.

13. Nardini M, Spanò S, Cericola C, Pesce A, Massaro A, Millo E, Luini A, Corda D, Bolognesi M: CtBP/BARS: a dual-function protein involved in transcription co-repression and Golgi membrane fission. EMBO J 2003, 22:3122-3130.

14. Stern MD, Aihara H, Cho KH, Kim GT, Horiguchi G, Roccaro GA, Guevara E, Sun HH, Neggri D, Tsukaya H, Nibu Y: Structurally related Arabidopsis ANGUSTIFORLIA is functionally distinct from the transcriptional corepressor CtBP. Dev Genes Evol 2007, 217:759-769.

15. Minamisawa N, Sato M, Cho K-H, Ueno H, Takechi K, Kajikawa M, Yamato KT, Ohyama K, Toyooka K, Kim G-T, Horiguchi G, Takano H, Ueda T, Tsukaya H: ANGUSTIFOLIA, a plant homolog of CtBP/BARS, functions outside the nucleus. Plant J 2011, 68:788-799.

16. Uhlrig JF, Mutondo M, Zimmermann I, Deeks MJ, Machesky LM, Thomas $P$, Uhrig S, Rambke C, Hussey PJ, Hulskamp M: The role of Arabidopsis SCAR genes in ARP2-ARP3-dependent cell morphogenesis. Development 2007, 134:967-977.

17. Zhang C, Mallery EL, Schlueter J, Huang S, Fan Y, Brankle S, Staiger CJ, Szymanski DB: Arabidopsis SCARs function interchangeably to meet actin-related protein $2 / 3$ activation thresholds during morphogenesis. Plant Cell 2008, 20:995-1011.

18. Yamaguchi-Shinozaki K, Shinozaki K: Arabidopsis DNA encoding two desiccation-responsive rd29 genes. Plant Physiol 1993, 101:1119-1120.

19. Kotchoni SO, Kuhns C, Ditzer A, Kirch H-H, Bartels D: Overexpression of different aldehyde dehydrogenase genes in Arabidopsis thaliana confers tolerance to abiotic stress and protects plants against lipid peroxidation and oxidative stress. Plant Cell Environ 2006, 29:1033-1048.

20. Wang C, Marshall A, Zhang D-B, Zoe A, Wilson ZA: ANAP: an integrated knowledge base for Arabidopsis protein interaction network analysis. Plant Physiol 2002, 158:1523-1533. 
21. Nibu Y, Zhang H, Bajor E, Barolo S, Small S, Levine M: dCtBP mediates transcriptional repression by Knirps, Kruppel and Snail in the Drosophila embryo. EMBO J 1998, 17:7009-7020.

22. Casson SA, Lindsey K: The turnip mutant of Arabidopsis reveals that LEAFY COTYLEDON1 expression mediates the effects of Auxin and sugars to promote embryonic cell identity. Plant Physiol 2006, 142:526-541.

23. Rossmann MG, Moras D, Olsen KW: Chemical and biological evolution of nucleotide-binding protein. Nature 1974, 250:194-199.

24. Schmitz F, Konigstorfer A, Sudhof TC: RIBEYE, a component of synaptic ribbons: a protein's journey through evolution provides insight into synaptic ribbon function. Neuron 2000, 28:857-872.

25. Zhang Q, Piston DW, Goodman RH: Regulation of corepressor function by nuclear NADH. Science 2002, 295:1895-1897.

26. Laloi C, Apel K, Danon A: Reactive oxygen signalling: the latest news. Curr Opin Plant Biol 2004, 7:323-328.

27. Barth C, Moeder W, Klessig DF, Conklin PL: The timing of senescence and response to pathogens is altered in the ascorbate-deficient Arabidopsis mutant vitamin c-1. Plant Physiol 2004, 134:1784-1792.

28. Kotchoni SO, Larrimore KE, Mukherjee M, Kempinski CF, Barth C: Alterations in the endogenous ascorbic acid content affect flowering time in Arabidopsis. Plant Physiol 2009, 149:803-815.

29. An Y-Q, McDowell JM, Huang S, McKinney EC, Chambliss S, Meagher RB: Strong, constitutive expression of the Arabidopsis ACT2/ACT8 actin subclass in vegetative tissues. Plant J 1996, 10:107-121.

30. Tiedemann AV: Evidence for a primary role of active oxygen species in induction of host cell death during infection of bean leaves with Botrytis cinerea. Physiol Mol Plant Pathol 1997, 50:151-166.

doi:10.1186/1471-2229-13-79

Cite this article as: Gachomo et al:: The cell morphogenesis

ANGUSTIFOLIA (AN) gene, a plant homolog of CtBP/BARS, is involved in abiotic and biotic stress response in higher plants. BMC Plant Biology 2013 13:79.

\section{Submit your next manuscript to BioMed Central and take full advantage of:}

- Convenient online submission

- Thorough peer review

- No space constraints or color figure charges

- Immediate publication on acceptance

- Inclusion in PubMed, CAS, Scopus and Google Scholar

- Research which is freely available for redistribution 